\title{
ASYMMETRIC ASPERITY HEIGHT DISTRIBUTIONS IN A SCALE-DEPENDENT MODEL FOR CONTACT AND FRICTION
}

\author{
George G. Adams \\ Department of Mechanical, Industrial and Manufacturing Engineering \\ Northeastern University, Boston, MA 02115, USA \\ Email: adams@neu.edu \\ Sinan Müftü
Department of Mechanical, Industrial and Manufacturing Engineering \\ Northeastern University, Boston, MA 02115, USA
}

\begin{abstract}
The effect of an asymmetric distribution of asperity heights is accounted for in a recently developed scale-dependent multiasperity model of contact and friction. A Weibull distribution of asperity heights is used which allows the skew and kurtosis to be varied, but not independently of each other. The contact and friction model used includes the effects of adhesion and of scale-dependent friction. The results obtained demonstrate that positive/negative skew decreases/increases both the friction coefficient and its dependence on the magnitude of the normal load.
\end{abstract}

\section{INTRODUCTION}

Contact and friction affect the operation of many machines and tools that we use every day, as well as some of the most basic activities in nature. Examples range from belt drives, brakes, tires, and clutches in automobiles and in other machines; gears, bearings and seals in a variety of mechanical systems; electrical contacts in motors; slider-disk interactions in a computer disk drive; various MEMS devices; a robotic manipulator joint; the motion of a human knee-joint (natural or artificial); and walking/running.

The friction force $F$ is the tangential force resisting the relative motion of two surfaces which are pressed against each other with a normal force $P$. Amontons, in 1699, and Coulomb in
1785, developed our phenomenological understanding of dry friction between two contacting bodies. Amontons-Coulomb friction states that the ratio of the friction force (during sliding) to the normal force is a constant called the coefficient of kinetic friction. Similarly the coefficient of static friction is the ratio of the maximum friction force $F$ that the surfaces can sustain, without relative motion, to the normal force. These friction laws can be summarized by defining the coefficient of friction $\mu$ as

$$
\mu=\frac{F}{P}
$$

without distinguishing between static and low-speed sliding friction. Although Eqn. (1) provides an extraordinarily simple phenomenological friction law, the nature of the friction force is not well-understood.

Contact modeling is an essential part of any friction model. It consists of two related steps. First, the equations representing the contact of a single pair of asperities are determined. For nanometer scale contacts the effect of adhesion on the contact area is important. Second, the cumulative effect of individual asperity contacts is determined. Such contact models are uncoupled and represent surface roughness as a set of asperities, often with statistically distributed parameters. The effect of each individual asperity contact is local and considered separately from the other asperities; the cumulative 
effect is the sum of the actions of individual asperities (e.g. the well-known Greenwood Williamson model, [1]).

For sufficiently small size contacts, the adhesion forces between the surfaces affect the contact conditions. Various adhesion models, typically between an elastic sphere and a flat, have been introduced. The model by Johnson, Kendall and Roberts (JKR) assumes that the attractive intermolecular surface forces cause elastic deformation beyond that predicted by the Hertz theory, thereby producing a subsequent increase of the contact area [2]. The model by Derjaguin, Muller and Toporov (DMT), on the other hand, accounts for the adhesive stress outside of the contact area, but assumes that the contact stress profile remains the same as in the Hertz theory [3]. Due to the assumptions involved, the JKR/DMT models are most suitable when the range of surface forces is small/large compared to the elastic deformations, as pointed out by Tabor [4]. Another model, introduced by Maugis [5], describes a continuous transition between the JKR and DMT models.

Contact and friction models which deal with adhesion in multi asperity contacts have also been developed. In the first of a series of papers Chang, Etsion and Bogy (CEB) [6] developed an elastic-plastic multiasperity contact model for normal loading based on volume conservation of a plastically deformed asperity control volume. In [7], the effect of adhesion was included by using the DMT model for contacting asperities and the Lennard-Jones potential between non-contacting asperities. Finally a model for calculating the coefficient of friction was given in [8]. It assumed that once plastic yielding is initiated in a pair of contacting asperities, no further tangential force can be sustained. Fuller and Tabor [9] investigated the effect of roughness on the adhesion between elastic bodies. Experiments were conducted between rubber spheres and a hard flat surface with controlled roughness. A theoretical model which used the JKR model of adhesion along with a Gaussian distribution of asperity heights was developed.

Stanley, Etsion and Bogy (SEB) [10] developed a model for the adhesion of two rough surfaces, affected by sub-boundary layer lubrication, in an elastic-plastic multi-asperity contact. Polycarpou and Etsion [11] used the SEB model to predict the static friction coefficient. The tangential load was found using the same procedure as in the CEB model [8] for solid-solid contact. Kogut and Etsion developed multi-asperity contact [12] and friction models [13] which included the effects of plastic deformation. The maximum shear load that an asperity can sustain is limited by the combined normal and shear load which causes the plastic deformation zone to reach the surface. Thus the friction analysis [13] predicts higher friction than the related work in [8].

The scale dependence of the friction stress for single asperity contacts has recently been investigated by Hurtado and Kim (HK), $[14,15]$. They presented a micromechanical dislocation model of frictional slip between two asperities for a wide range of contact radii. According to the HK model, if the contact radius " $a$ " is smaller than a critical value, the asperities slide past each other in a concurrent slip process where the adhesive forces are responsible for the shear stress; hence the shear stress remains at a high constant value. On the other hand, if the

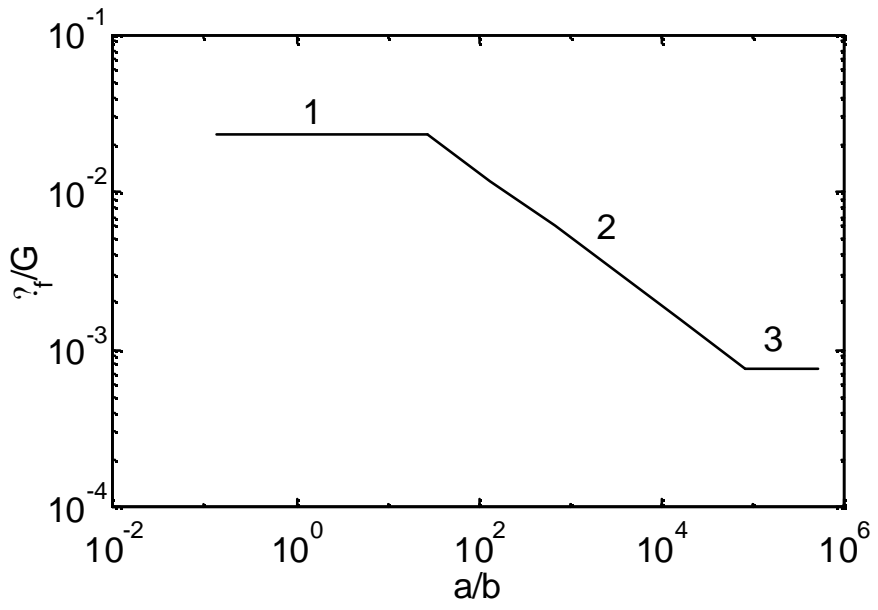

Figure 1. Relationship between friction stress and contact radius according to $\mathrm{HK}$ model.

contact radius is greater than that critical value, the shear stress decreases for increasing values of contact radius until it reaches a second constant, but lower value. The relationship between the non-dimensional friction stress $\left(\bar{\tau}_{f}=\tau_{f} / G^{*}\right)$ and the non-dimensional contact radius $(\bar{a}=a / b)$ is approximated in Fig. 1. The contact radius " $a$ " is normalized by the Burgers vector $b$ and the friction stress $\tau_{f}$ is normalized by the effective shear modulus $G^{*}=2 G_{1} G_{2} /\left(G_{1}+G_{2}\right)$ where $G_{1}$ and $G_{2}$ are the shear moduli of the contacting bodies. Adams, Müftü, and Mohd Azhar (AMM), [16] incorporated the HK model and the adhesion contact model of Maugis, into a statistical multi asperity model for contact and friction. The relationship between the friction force and the normal load between two rough surfaces during a slip process was determined. Three key dimensionless parameters representing the surface roughness, the friction regime of the contacts, and the surface energy of adhesion, were seen to influence the value of the friction coefficient.

The previously cited works used a Gaussian distribution of asperity heights. The effect of asymmetry in the height distribution has been accounted for by a few authors. Kotwal and Bhushan [17] modeled this effect and found that there are load-dependent optimal values of skew and kurtosis which minimize the real area of contact. McCool [18] extended the GW model by using a Weibull distribution of asperity heights and by including the effect of a surface coating. The multiasperity elastic-plastic CEB contact model was generalized to include a Weibull distribution by $\mathrm{Yu}$ and Polycarpou [19]. Skew was found to greatly affect the relation between the force, contact area, and the number of contacting asperities

In this paper the effect of an asymmetric distribution of asperity heights is accounted for in the recently developed AMM scaledependent multi-asperity model of contact and friction. A Weibull distribution of asperity heights is used which allows the skew and kurtosis to be varied, but not independently of each other. The contact and friction model used includes the effects of adhesion and of scale-dependent friction. The results demonstrate that positive/negative skew decreases/increases 
both the friction coefficient and its dependence on the magnitude of the normal load.

\section{DEVELOPMENT OF THE ASYMMETRIC MODEL Contact Model}

The scale-dependent multi-asperity contact and friction model developed by Adams, Müftü, and Mohd Azhar [16] will be extended to include asymmetric asperity height distributions. For two real surfaces separated by a distance $d$ (defined from the mean of asperity heights) the number of contacting asperities $n$ is

$$
n=N \int_{\bar{d}}^{\infty} \bar{\phi}(\bar{z}) d \bar{z}
$$

where $N$ is the total number of asperities, $\sigma$ is the standard deviation of asperity peak heights, $\bar{z}=z / \sigma$ is the dimensionless height coordinate measured from the mean of asperity heights, $\bar{\phi}(\bar{z})$ is the probability density of asperity peaks, and $\bar{d}=d / \sigma$ is the non-dimensional separation between the two surfaces (Fig. 2).

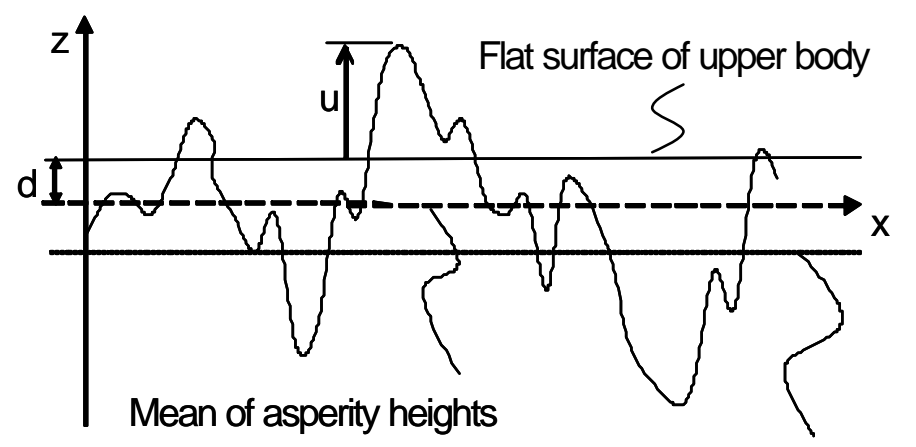

Mean of surface heights

Figure 2. Contact of a rough surface (lower body) with a flat surface (upper body).

The relation between the normal load $P$ and deformation $u=z-d$ of two contacting spherical asperities with adhesion is given by the Maugis model [5]. In that model, a uniform tensile stress $\sigma_{0}$ exists between the contacting asperities just outside the contact zone, $a \leq r \leq c$, where $c$ is the radial extent of the adhesion zone. The separation between the two surfaces at $r=c$ is equal to the prescribed maximum adhesion distance $h$. Thus the work of adhesion is given by $w=\sigma_{0} h$. In [5] the following non-dimensional relations among the asperity contact radius $\left(A_{M}\right)$, the asperity contact force $\left(P_{M}\right)$, and the asperity deformation $\left(\Delta_{M}\right)$ were obtained

$$
\begin{aligned}
& \frac{? A_{M}^{2}}{2}\left[\sqrt{m^{2}-1}+\left(m^{2}-2\right) \tan ^{-1} \sqrt{m^{2}-1}\right] \\
& +\frac{4 ?^{2} A_{M}}{3}\left[\sqrt{m^{2}-1} \tan ^{-1} \sqrt{m^{2}-1}-m+1\right]=1
\end{aligned}
$$

$$
\begin{gathered}
P_{M}=A_{M}{ }^{3}-\lambda A_{M}{ }^{2}\left[\sqrt{m^{2}-1}+m^{2} \tan ^{-1} \sqrt{m^{2}-1}\right] \\
\Delta_{M}=A_{M}{ }^{2}-\frac{4}{3} A_{M} \lambda \sqrt{m^{2}-1}
\end{gathered}
$$

where $\lambda$ is the non-dimensional Maugis adhesion parameter, and $m$ is the non-dimensional adhesion radius. The various non-dimensional quantities used in the Maugis model are defined as

$$
\begin{aligned}
& A_{M}=a\left(\frac{K}{\pi w R^{2}}\right)^{1 / 3}, \quad \lambda=2 \sigma_{0}\left(\frac{R}{\pi w K^{2}}\right)^{1 / 3}, \\
& m=\frac{c}{a}, \quad P_{M}=\frac{P}{\pi w R}, \quad \Delta_{M}=u\left(\frac{K^{2}}{\pi^{2} w^{2} R}\right)^{1 / 3}
\end{aligned}
$$

where $K=(4 / 3) E^{*}$.

The simultaneous solution of Eqs. (3)-(5) gives the relations between $m, P_{M}, A_{M}$ and $\Delta_{M}$ for a given value of $\lambda$. In practice it is convenient to vary $m$ and solve the resulting quadratic equation for the only positive root $A_{M}$ in (3). Then (4) and (5) can be solved explicitly for $P_{M}$ and $\Delta_{M}$ respectively. The nondimensional Maugis adhesion parameter $\lambda$ is expressed in terms of the surface roughness parameter $\alpha$, the friction regime parameter $\beta$, the surface energy of adhesion parameter $\gamma$, the maximum adhesion distance $h$, and the Burgers vector $b$, as

$$
\lambda=\left(\frac{b}{h}\right)\left(\frac{9 \beta \gamma^{2}}{2 \pi \alpha}\right)^{1 / 3}
$$

where the three key parameters $\alpha, \beta$ and $\gamma$ are

$$
\alpha=\left(\frac{\sigma}{R}\right)^{1 / 2}, \quad \beta=\frac{(R \sigma)^{1 / 2}}{b}, \quad \gamma=\frac{w}{E^{*} b}
$$

A physical interpretation of the surface parameters $\alpha$ and $\beta$ is provided by noting that in a simple vertical scaling of the surface by a factor $k$, the standard deviation of asperity heights $\sigma$ is scaled by $k$ but the asperity radius of curvature $R$ is scaled by $1 / k$. Thus, $\alpha$ is scaled by $k$, but $\beta$ remains constant. Hence $\alpha$ is a representation of the surface roughness, and is referred to as the surface roughness parameter. The parameter $\beta$ describes the ratio of the contact radius (due to an asperity penetration equal to $\sigma$ ) to the Burgers vector length. Thus small $\beta$ are expected to be indicative of nano-scale asperity contacts and progressively larger values of $\beta$ correspond to transition and larger values of the contact radius (Fig. 2). Therefore $\beta$ is referred to as the friction regime parameter. The surface energy parameter $\gamma$ represents the ratio of the adhesive stress to the product of the composite Young's modulus and the Burgers vector. 
It is further noted that for the case considered here, in which one of the surfaces is assumed to be rigid and flat, $G^{*}=2 G$ and the composite Young's modulus is given by $E^{*}=E /\left(1-v^{2}\right)$. Furthermore the relation $G=E / 2(1+v)$ has been used in obtaining (7).

The non-dimensional contact radius, normal load, and asperity penetration used in the Maugis model $\left(A_{M}, P_{M}\right.$ and $\Delta_{M}$, respectively) can be expressed in terms of the corresponding non-dimensional parameters defined in this work $(\bar{a}, \bar{P}, \bar{u}=u / \sigma$, respectively) according to

$$
\bar{u}=\Delta_{M} \hat{\delta}, \quad \bar{P}=P_{M} / \hat{P}, \quad \bar{a}=A_{M} / \hat{A}
$$

where

$$
\hat{\delta}=\left(\frac{3 \pi \gamma}{4 \alpha^{2} \beta}\right)^{2 / 3}, \quad \hat{P}=\frac{(1-v) \alpha^{2}}{2 \pi \beta \gamma}, \hat{A}=\left[\frac{4}{3 \pi \gamma}\left(\frac{\alpha}{\beta}\right)^{2}\right]^{1 / 3} \text {. }
$$

An expression for the non-dimensional normal force $\bar{P}$ acting on the nominal contact area is obtained by integrating the normal force on individual asperities from the Maugis model $\left(P_{M}\right)$, i.e.

$$
\bar{P}=\frac{1}{\hat{P}} \int_{\bar{d}}^{\infty} P_{M} \phi(\bar{z}) d \bar{z}
$$

It is noted that due to adhesion, asperities may come into contact even if the asperity overlap $u$ is negative. This effect has been included in the evaluation of the integrals in Eqs. (2) and (10) by varying the adhesion radius ratio $m$ in Eqs. (3)-(5) when evaluating the force and contact area. Thus the lower limits of the integrals in Eqs. (2) and (10) are effectively slightly less than $\bar{d}$.

\section{Friction Model}

Although adhesion affects the relationship between the normal force and the contact radius, it does not affect the relation between the friction force and contact radius. From Fig. 1, the dimensionless shear stress is a function of the dimensionless contact radius and is approximated by

$$
\log \left(\bar{\tau}_{f}\right)=\left\{\begin{array}{cl}
\log \bar{\tau}_{f_{1}}, & \bar{a}<\bar{a}_{1} \\
M \log \bar{a}+B, & \bar{a}_{1}<\bar{a}<\bar{a}_{2} \\
\log \bar{\tau}_{f_{2}}, & \bar{a}>\bar{a}_{2}
\end{array}\right.
$$

where the left and right limits of region-2 are $\left(\bar{a}_{1}, \bar{\tau}_{f_{1}}\right)$ and $\left(\bar{a}_{2}, \bar{\tau}_{f_{2}}\right)$ respectively. The constants of Eq. (11) are given by

$$
\begin{aligned}
M & =-\left(\log \left(\bar{\tau}_{f_{1}} / \bar{\tau}_{f_{2}}\right)\right) /\left(\log \left(\bar{a}_{2} / \bar{a}_{1}\right)\right) \\
B & =\left(\log \left(\bar{\tau}_{f_{1}}\right) \log \left(\bar{a}_{2}\right)-\log \left(\bar{\tau}_{f_{2}}\right) \log \left(\bar{a}_{1}\right)\right) /\left(\log \left(\bar{a}_{2} / \bar{a}_{1}\right)\right)
\end{aligned}
$$

where $M$ and $B$ are, respectively, the slope and $y$-intercept of the line in region-2 of the log-log plot of Fig. 1. The friction force $F_{f}$ acting on a single asperity can be determined from Eq. (11) by using $F_{f}=\pi a^{2} \tau_{f}$ resulting in

$$
\frac{F_{f}}{G^{*} b^{2}}=\left\{\begin{array}{cl}
\bar{\tau}_{f_{1}} \bar{a}^{2}, & \bar{a}<\bar{a}_{1} \\
10^{B} \bar{a}^{M+2}, & \overline{a_{1}}<\bar{a}<\bar{a}_{2} \\
\bar{\tau}_{f_{2}} \bar{a}^{2}, & \bar{a}>\bar{a}_{2}
\end{array}\right.
$$

The total shear force $F$ acting on the nominal contact area can be calculated by integrating the shear forces acting on each asperity against the probability density function, i.e.

$$
F=N \int_{\bar{d}}^{\infty} F_{f} \phi(\bar{z}) d \bar{z} .
$$

It is noted that for values of the applied tangential force $F$ less than that given by Eq. (14), the distribution of tangential and normal forces among the asperities may cause some asperities to slip while others continue to stick. However when $F$ reaches the value given in Eq. (14) all contacting asperities will slide resulting in global slip. In non-dimensional form, the force per asperity $(\bar{F})$ becomes, using Eqs. (13-14)

$$
\begin{aligned}
\bar{F}= & \frac{F}{N G b^{2}}=2 \pi \int_{\bar{d}}^{\bar{z}_{1}} \bar{\tau}_{f_{1}} \bar{a}^{2} \phi(\bar{z}) d \bar{z}+ \\
& 2 \pi \int_{\bar{z}_{1}}^{\bar{z}_{2}} 10^{B} \bar{a}^{M+2} \phi(\bar{z}) d \bar{z}+2 \pi \int_{\bar{z}_{2}}^{\infty} \bar{\tau}_{f_{2}} \bar{a}^{2} \phi(\bar{z}) d \bar{z}
\end{aligned}
$$

Thus, the coefficient of friction $\mu$ for two real surfaces separated by a distance $\bar{d}$, can be obtained from Eqs. (1), (10), and (15).

\section{Weibull Distribution}

In [16] a Gaussian distribution of asperities heights was assumed. In this paper we investigate the effect of asymmetry by using a Weibull function, i.e.

$$
\phi(\hat{z})=\frac{\omega \hat{z}^{\omega-1}}{\eta^{\omega}} e^{-(\hat{z} / \eta)^{\omega}}, \quad \hat{z}>0
$$

to represent the asperity height distribution. For this twoparameter Weibull function the mean $\left(z_{M}\right)$, standard deviation of asperity heights $(\sigma)$, skew $(S)$, and kurtosis $(K)$ are given by in terms of the two Weibull parameters $(\omega, \eta),[20]$.

The Weibull distribution variable $(\hat{z})$ is then shifted by 


$$
z=\hat{z}-z_{M}, \quad z_{M}=\eta B_{1}, \quad B_{n}=\Gamma\left(1+\frac{n}{\omega}\right),
$$

where $\Gamma$ is the gamma function, so that $z=0$ corresponds to the mean of asperity heights.

Furthermore using the non-dimensional variable $(\bar{z}=z / \sigma)$

$$
\bar{\phi}(\bar{z})=\omega C^{\omega} \bar{z}^{\omega-1} e^{-(C \bar{z})^{\omega}}, \quad C=\left(B_{2}-B_{1}^{2}\right)^{1 / 2}
$$

is obtained. Note that this form of the Weibull distribution depends on only one parameter $(\omega)$ and that $\bar{z}=0$ corresponds to the mean of asperity heights. The skew, standard deviation, and kurtosis are given by

$$
\begin{aligned}
& S=\left(B_{3}-3 B_{2} B_{1}+2 B_{1}^{3}\right) / C^{3}, \\
& \sigma=\eta C \\
& K=\left(B_{4}-4 B_{3} B_{1}+6 B_{2} B_{1}^{2}-3 B_{1}^{4}\right) / C^{4}
\end{aligned}
$$

respectively. It follows that these non-dimensional quantities also depend only on the parameter $\omega$. As $\omega$ is varied both the skew and kurtosis change. However it is not possible to change the skew without also changing the kurtosis as indicated by Eqn. (19).

\section{RESULTS AND DISCUSSION}

Results have been obtained for the friction coefficient as a function of the normal load, for various values of the skew $(\bar{S})$ and the three key parameters $(\alpha, \beta, \gamma)$ defined in the AMM model. Figure 3 shows the effect of skew for the default values $(\alpha=0.01, \beta=1000, \gamma=0.001)$ used in [16]. Positive/negative skew is seen to decrease/increase both the friction coefficient as well as its variation with normal load. This result is due to negative skew causing more asperities to be above the mean of asperity heights than below. Correspondingly there is less of a variation in height distribution for the $\mathrm{z}>0$ portion than for $\mathrm{z}<0$. The more lightly loaded the contact, the smaller is the fraction of the Weibull distribution which is in contact. Thus the asymmetry in the height distribution causes the surface to appear as if its roughness is reduced for lightly loaded contacts .

Figures 4, 5, and 6 show similar results for a lower value of the surface roughness parameter $(\alpha=0.006)$, for a lower value of the friction regime parameter $(\beta=500)$, and for a higher value of the adhesion parameter $(\gamma=0.002)$ respectively. The smoother surface shows a greater effect of skew than the rougher one. This result may be expected based on the previous discussion. The effect of a reduction of the friction regime parameter is to increase friction for the Gaussian distribution. However as the skew decreases to the value of -1 the effect of $\beta$ on friction decreases. The effect of an increase in the surface energy parameter is to increase friction for both positive and negative skew values.

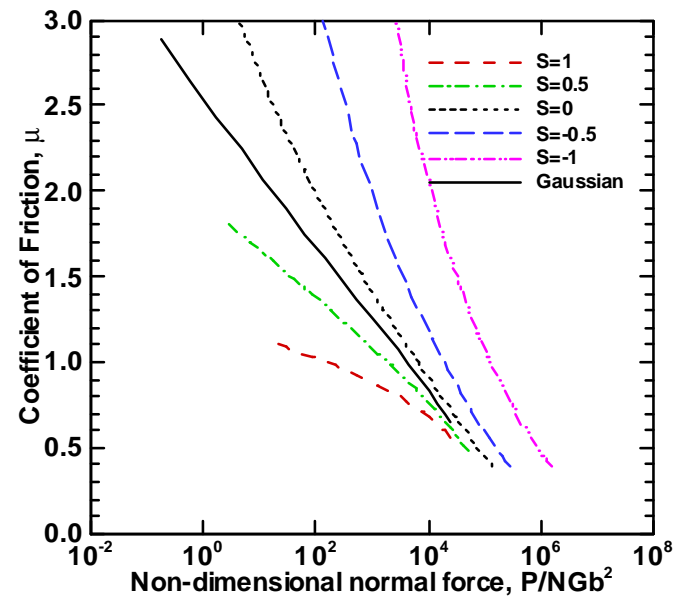

Figure 3. Coefficient of friction vs. normal load for various skew and $\alpha=0.01, \beta=1000, \gamma=0.001$

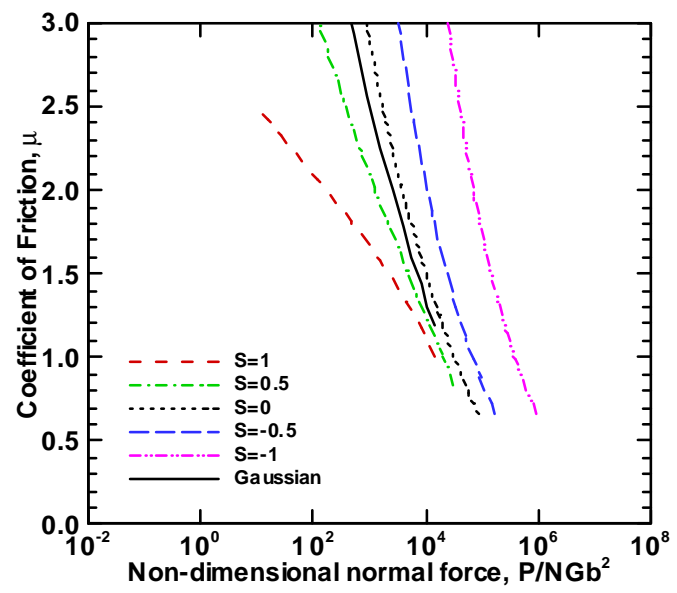

Figure 4. Coefficient of friction vs. normal load for various skew and $\alpha=0.006, \beta=1000, \gamma=0.001$

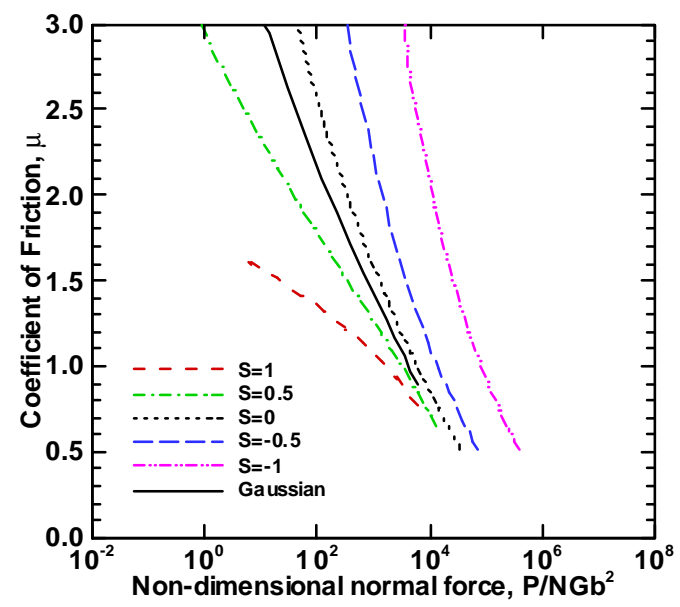

Figure 5. Coefficient of friction vs. normal load for various skew and $\alpha=0.01, \beta=500, \gamma=0.001$ 


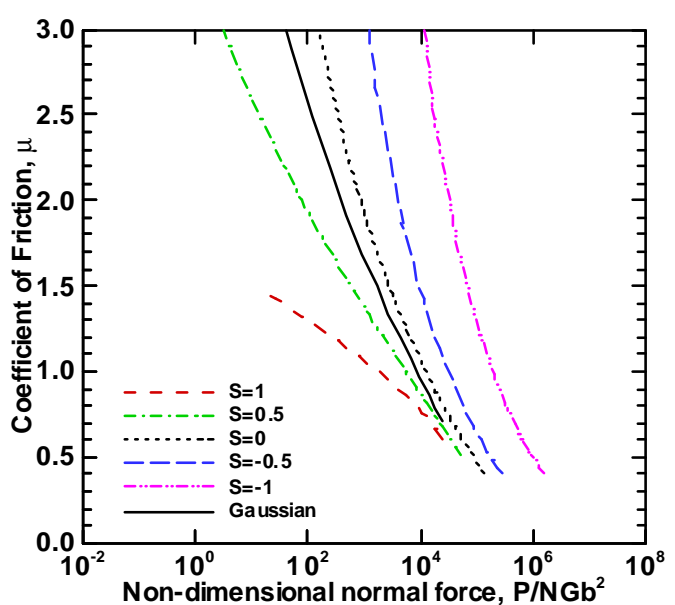

Figure 6. Coefficient of friction vs. normal load for various skew and $\alpha=0.01, \beta=1000, \gamma=0.002$

It is noted that the friction results for the Gaussian distribution do not correspond exactly to the zero skew case of the Weibull distribution. This difference is because the Weibull distribution only approximates a Gaussian distribution. For lightly loaded contacts the difference between these two distributions in their "leading edge" is sufficient to produce these discrepancies. The values of the Weibull shape parameter $(\omega)$, the mean $\left(\bar{z}_{M}\right)$ the skew $(\bar{S})$, and kurtosis $(\bar{K})$ are shown in Table 1 below.

\begin{tabular}{|c|c|c|c|}
\hline $\begin{array}{c}\text { Parameter } \\
\omega\end{array}$ & Mean & Skew & Kurtosis \\
$\bar{z}_{M}$ & $\bar{S}$ & $\bar{K}$ \\
\hline 40.74 & 32.32 & -1 & 4.773 \\
\hline 7.493 & 6.340 & -0.5 & 3.258 \\
\hline 3.602 & 3.243 & 0.0 & 2.716 \\
\hline 2.216 & 2.098 & 0.5 & 3.028 \\
\hline 1.564 & 1.530 & 1.0 & 4.159 \\
\hline
\end{tabular}

\section{CONCLUSIONS}

The effect of an asymmetric distribution of asperity heights has been accounted for in the recently developed scale-dependent multi-asperity model of contact and friction of Adams, Müftü and Mohd Azhar [16]. A Weibull distribution of asperity heights was used which allowed the skew and kurtosis to be varied, but not independently of each other. The contact and friction model included the effects of adhesion and of scaledependent friction. The results obtained demonstrated that positive/negative skew decreases/increases both the friction coefficient and its dependence on the magnitude of the normal load.

\section{REFERENCES}

[1] Greenwood, J.A., and Williamson, J.B.P., 1966, "Contact of Nominally Flat Surfaces," Proceedings of the Royal Society of London, A295, pp. 300-319.
[2] Johnson, K.L., Kendall, K., and Roberts, A.D., 1971, "Surface Energy and the Contact of Elastic Solids," Proceedings of the Royal Society of London, A324, pp. 301313.

[3] Derjaguin, B.V., Muller, V.M., and Toporov, Y.P., 1975, "Effect of Contact Deformations on the Adhesion of Particles," Journal of Colloid and Interface Science, 53, pp. 314-326.

[4] Tabor, D., 1976, "Surface Forces and Surface Interactions," Journal of Colloid and Interface Science, 58, pp. 2-13.

[5] Maugis, D., 1992, "Adhesion of Spheres: The JKR-DMT Transition Using a Dugdale Model," Journal of Colloid and Interface Science, 150, pp. 243-269.

[6] Chang, R.W., Etsion, I., Bogy, D.B., 1987, "An ElasticPlastic Model for the Contact of Rough Surfaces," ASME Journal of Tribology, 109, pp. 257-263.

[7] Chang, R.W., Etsion, I., Bogy, D.B., 1988, "Adhesion Model for Metallic Rough Surfaces," ASME Journal of Tribology, 110, pp. 50-56.

[8] Chang, R.W., Etsion, I., Bogy, D.B., 1988, "Static Friction Coefficient Model for Metallic Rough Surfaces" ASME Journal of Tribology, 110, pp. 57-63.

[9] Fuller, K.N.G and Tabor, D., 1975, "The Effect of Surface Roughness on the Adhesion of Elastic Solids," Proceedings of the Royal Society of London, A345, pp. 327342.

[10] Stanley, H.M., Etsion, I. and Bogy, D.B., 1990, "Adhesion of Contacting Rough Surfaces in the Presence of Sub-Boundary Lubrication," ASME Journal of Tribology, 112 , pp. 98-104.

[11] Polycarpou, A.A., and Etsion, I., 1998, "Static Friction of Contacting Real Surfaces in the Presence of Sub-Boundary Lubrication," ASME Journal of Tribology, 120, pp. 296-303.

[12] Kogut, L., and Etsion, I., 2003, "A Finite Element Based Elastic-Plastic Model for the Contact of Rough Surfaces," Tribology Transactions, 46, pp. 383-390.

[13] Kogut, L., and Etsion, I., 2003, "A Static Friction Model for Elastic-Plastic Contacting Rough Surfaces," ASME Journal of Tribology, in press.

[14] Hurtado, J.A,. and Kim, K.-S., 1999, "Scale Effects in Friction of Single Asperity Contacts: Part I; From Concurrent Slip to Single-Dislocation-Assisted Slip," Proceedings of the Royal Society of London, A455, pp. 3363-3384.

[15] Hurtado, J.A., and Kim, K.-S.,1999, "Scale Effects in Friction in Single Asperity Contacts: Part II; MultipleDislocation-Cooperated Slip," Proceedings of the Royal Society of London, A455, pp. 3385-3400.

[16] Adams, G.G., Müftü, S., and Mohd Azhar, N., 2003, "A Scale-Dependent Model for Multi-Asperity Model for Contact and Friction," ASME Journal of Tribology, 125, in press.

[17] Kotwal, C.A., and Bhushan, B., 1996, "Contact Analysis of Non-Gaussian Surfaces for Minimum Static and Kinetic Friction and Wear," Tribology Transactions, 39, pp. 890-898.

[18] McCool, J.I., 2000, "Extending the Capability of the Greenwood Williamson Microcontact Model," ASME Journal of Tribology, 122, pp. 496-502.

[19] Yu, N., and Polycarpou, A.A., 2002, "Contact of Rough Surfaces With Asymmetric Distribution of Asperity Heights," ASME Journal of Tribology, 124, pp. 367-376.

[20] Johnson, N., and Kotz, S., 1970, Continuous Univariate Distributions-I, John Wiley and Sons, Inc., New York. 